\title{
GATAD2B Gene Microdeletion Causing Intellectual Disability Autosomal Dominant Type 18: Case Report and Review of the Literature
}

\author{
Milana Trubnykova ${ }^{a}$ Jeny Bazalar Montoya ${ }^{b}$ Jorge La Serna-Infantes ${ }^{c}$ \\ Flor Vásquez Sotomayor ${ }^{a}$ María del Carmen Castro Mujica ${ }^{d}$ \\ Hugo Hernán Abarca Barrigaa, d, e \\ a Servicio de Genética \& Errores Innatos del Metabolismo (EIM), Instituto Nacional de Salud del Niño-Breña, \\ bUnidad de Genética \& Biología Molecular, Instituto Nacional de Enfermedades Neoplásicas, 'Servicio de \\ Citogenética \& Citopatología, Hospital Nacional Guillermo Almenara Irigoyen, d Facultad de Medicina Humana, \\ Universidad Ricardo Palma, and eFacultad de Odontología, Universidad Científica del Sur, Lima, Peru
}

\section{Keywords}

Chromosomal microarray analysis - Copy number variation . GATAD2B $\cdot$ Intellectual disability $\cdot$ MRD18

\footnotetext{
Abstract

Pathogenic variants of the GATAD2B gene (1q21.3) are linked to intellectual disability autosomal dominant type 18 (MRD18; MIM 615074), characterized by dysmorphic features, psychomotor and language delay. We present an 11-year-old female patient with intellectual disability and typical clinical characteristics of MRD18. Chromosomal microarray analysis (CMA) revealed a novel CNV, approximately $200 \mathrm{~kb}$ in size and showed that the INTS3 and SLC27A3 genes are completely deleted along with the first 10 exons of the GATAD2B gene. INTS3 encodes the integrator complex subunit 3 and is part of the complex that maintains genome stability; SLC27A3 encodes a fatty acid transporter and has been associated with autism spectrum disorder. GATAD2B haploinsufficiency is associated with the phenotype. Furthermore, the girl had other clinical characteristics not previ-
}

ously described, such as emotional instability, calf hypotrophy, hypoplastic digit pads, tapered thumbs, and anterior earlobe crease. This study highlights the importance of the phenotype-genotype correlation using molecular diagnostic techniques, such as CMA, and its impact on precise diagnosis, treatment, prognosis, and genetic counseling for patients and their families.

(c) 2019 S. Karger AG, Basel

The Diagnostic and Statistical Manual of Mental Disorders (DSM-5) classifies intellectual disability (ID) as a generalized neurodevelopment disorder characterized by significant limitations in intellectual functioning (IQ $<70$ ) and adaptive behavior (including conceptual, social, and practical skills) under 18 years of age. ID, either isolated or syndromic, affects $1-8 \%$ of the worldwide population (alone $3-12.9 \%$ in the Latin American population) [Ropers, 2010; Lazcano-Ponce et al., 2013; Srivastava and Schwartz, 2014; Vissers et al., 2016].

\section{KARGER}

(c) 2019 S. Karger AG, Basel

E-Mail karger@karger.com

www.karger.com/msy
Hugo Hernán Abarca Barriga

Instituto Nacional de Salud del Niño-Breña, Servicio de Genética \& EIM Av. Brasil 600

Breña, Lima 15083 (Peru)

E-Mail habarca@insn.gob.pe 
Fig. 1. Pedigree of the family showing the proband III.2 (arrow) and her maternal aunt affected by scleroderma (II.4).

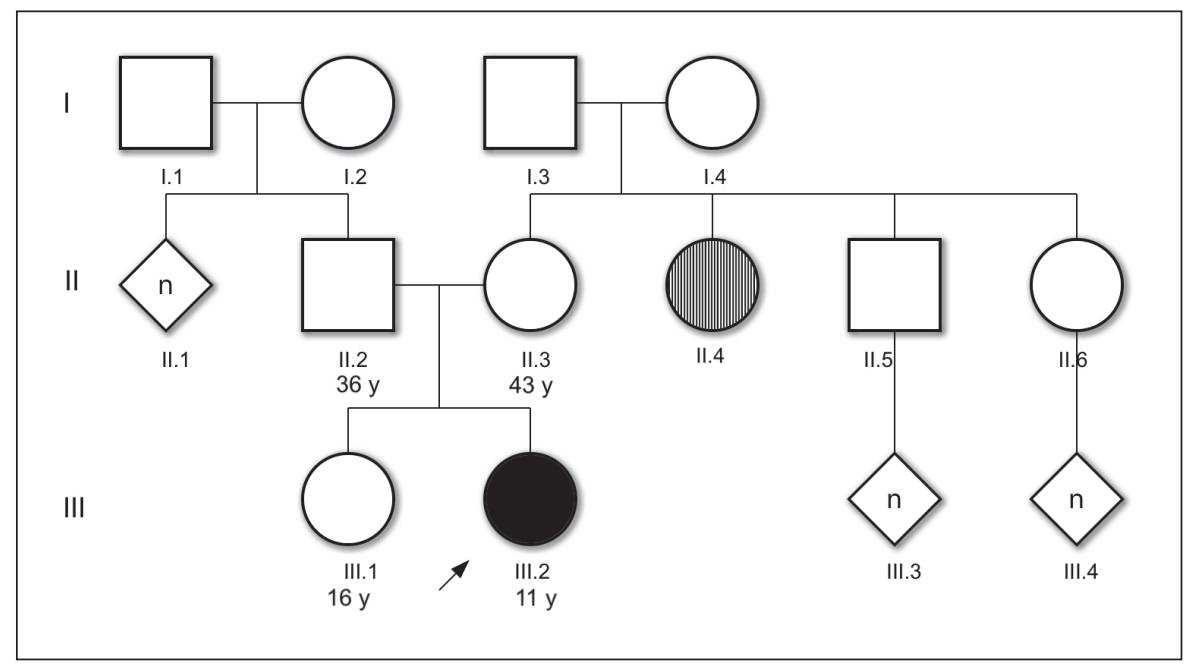

Normal brain development depends on a precise and complex sequence of events such as proliferation, migration, connection, and neuronal-glial maturation. Even in a morphologically normal brain, neuronal connectivity can be altered by glial or synaptic dysfunction, or accumulation of toxic components [Chiurazzi and Pirozzi, 2016].

ID can be caused by genetic and/or environmental factors beginning in the embryonic period until adulthood. ID has a high degree of clinical and genetic heterogeneity. Currently, some studies have estimated that up to $90 \%$ of the ID cases are caused by genetic variants and are diagnosed by modern molecular techniques such as chromosomal microarray analysis (CMA). Genetic causes account for $25 \%$ of all the patients, and in males with ID, $\mathrm{X}$-linked genes are responsible for 10-12\% [Ropers, 2010; Srivastava and Schwartz, 2014; Karam et al., 2015; Vissers et al., 2016].

In the Online Mendelian Inheritance in Man (OMIM; www.omim.org), ID has been classified into autosomal dominant (PS156200, 50 types), autosomal recessive (PS249500, 53 types), syndromic X-linked (PS309510, 41 types), and nonsyndromic X-linked (PS309530, 50 types). It is important to discriminate between syndromic and isolated types; however, some genetic variants may also cause syndromic and isolated ID. Several studies demonstrate genetic overlap between ID and other neuropsychiatric disorders [Chiurazzi and Pirozzi, 2016; Vissers et al., 2016).

The GATA zinc finger domain containing $2 \mathrm{~B}$ gene (GATAD2B; MIM 614998; 1q21.3) encodes a protein transcriptional repressor p66-beta (p66 $\beta$; Uniprot
Q8WXI9). GATAD2B belongs to the MeCP1 complex (methyl-CpG-binding domain protein 1) and its variants are related to intellectual disability autosomal dominant 18 (MRD18; MIM 615074) [de Ligt et al., 2012; Willemsen et al., 2013; Hamdan et al., 2014; Luo et al., 2017; TimAroon et al., 2017; Vermeulen et al., 2017].

We report the first Peruvian female patient, and 16th worldwide, with a molecular diagnosis of MRD18 revealed by CMA.

\section{Clinical Report}

Our female patient was born at term by vaginal delivery after an uneventful pregnancy to nonconsanguineous parents born in Ancash, a suburban region of Peru. The girl's birth weight was $3,500 \mathrm{~g}$ (58th percentile). Apgar score head circumference, and height were not documented. Her father was 25 years old and her mother was 32 when the child was born. Medical history reported pneumonia at 6 months and strabismus at 2 years of age; her maternal aunt has scleroderma (Fig. 1). Regarding her developmental milestones, she was able to hold her head independently at 4 months, sit without support at 7 months, walk independently at 18 months, and she spoke her first word at 14 months. She was referred to our institute for genetic evaluation due to speech delay and ID. Currently, she is in third grade at primary school with poor performance.

At physical examination (11 years), anthropometric measurements were: weight $29.7 \mathrm{~kg}$ (10th percentile), height $139 \mathrm{~cm}$ (16th percentile), and head circumference $53.5 \mathrm{~cm}$ (30th percentile). The phenotype showed dysmorphic features characterized by a long face, thin brown hair, a broad forehead, hypoplastic superciliary arches, eyebrows laterally not extended, long tubular nose with bulbous nasal tip, upslanting palpebral fissures, malar hypoplasia, full cheeks, short philtrum, thin lip vermillion, prognathism, and anterior earlobe crease. Musculoskeletal abnormalities included 
Fig. 2. Clinical characteristics. a, b Long face, thin brown hair, a broad forehead, hypoplastic superciliary arches, eyebrows laterally not extended, long tubular nose with bulbous nasal tip, upslanting palpebral fissures, malar hypoplasia, prominent cheeks, short philtrum, thin lip vermillion, and prognathism. c Scoliosis. d, e, k, I Underdeveloped distal interphalangeal crease of the fourth finger, and tapered digits. $\mathbf{f ~ H y - ~}$ poplastic digit pads. $\mathbf{g}, \mathbf{h}$ Anterior earlobe crease. i, j Calf hypotrophy. $\mathbf{m}$ Café-au-lait spot in the lumbar region.
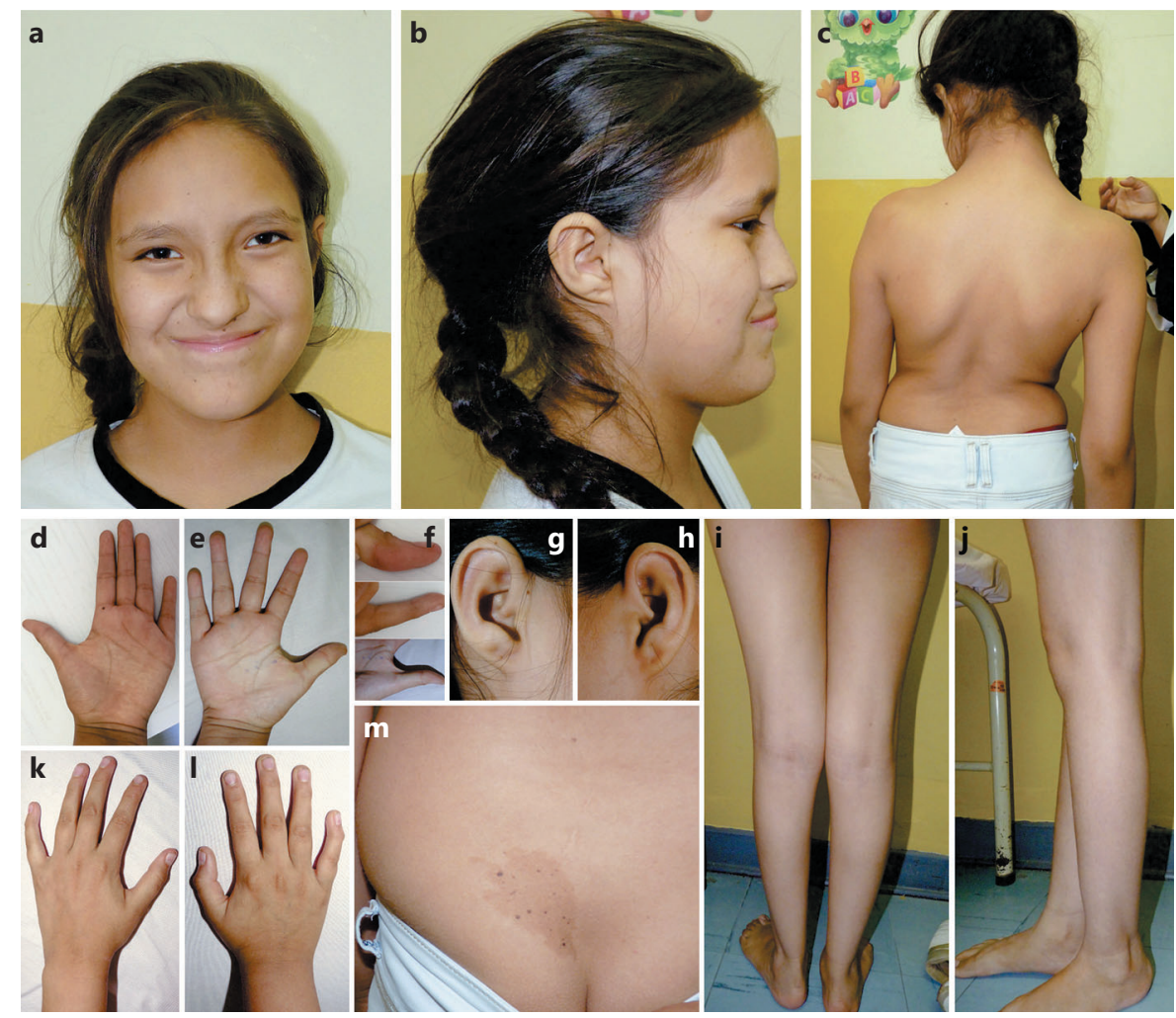

an elongated chest, scoliosis, hypoplastic digit pads, underdeveloped distal interphalangeal crease of the fourth finger, tapered thumbs, flat feet, and calf hypotrophy. Furthermore, she showed a café-au-lait spot with an irregular border in the lumbar region and inappropriate laughter (Fig. 2).

Our patient had an IQ of 39 (moderate ID), chromosome analysis showed a normal karyotype (46,XX), X-rays detected dorsal and lumbar rotoscoliosis, EEG revealed frontoparietal intermittent alpha activity, and thin layer chromatography of amino acids from urine were normal.

\section{Methods}

CMA from total DNA (250 ng) was performed according to international recommendations [Miller et al., 2010], amplified, labeled, and hybridized using GeneChip CytoScan 750K Array protocols (Affymetrix, Santa Clara, CA, USA) according to the manufacturer's instructions. The array specifications included 550,000 non-polymorphic markers and 200,436 SNP markers. CEL files obtained by scanning the arrays were analyzed using the Chromosome Analysis Suite (ChAS) software (Affymetrix). Gains and losses that affected a minimum of 25/50 markers, respectively, and loss-of-heterozygosity regions that expanded over $5 \mathrm{Mbp}$ were initially considered.

The patient's CNVs were compared with genomic variants in public databases, including DECIPHER [Firth et al., 2009] and UCSC (University of California, Santa Cruz) Genome Browser.

\section{Results}

CMA revealed a heterozygous deletion approximately $200 \mathrm{~kb}$ in size (arr[hg19] 1:153667997_153867908) in the long arm of chromosome 1 (1q21.3), which was classified as pathogenic CNV. This deletion encompassed 3 genes and involves the total loss of INTS3 and SLC27A3, which are not described in OMIM. In addition, CMA detected a partial deletion (first 10 exons) of the GATAD2B gene, which is known to be related to MRD18 (Fig. 3). She did not show other pathogenic or likely pathogenic variants. Loss of heterozygosity showed $1.26 \%$. CMA was not performed for the parents.

\section{Discussion}

We described a female patient with moderate ID, language delay, and dysmorphic features. More than 1,000 variants were found in clinical databases (Face2Gene ${ }^{\circledR}$, Phenomizer $^{\circledR}$, and OMIM) according to this phenotype; hence, the clinical diagnosis was impossible. However, using massive molecular technologies such as CMA, a partial deletion of the GATAD $2 B$ gene was revealed, 


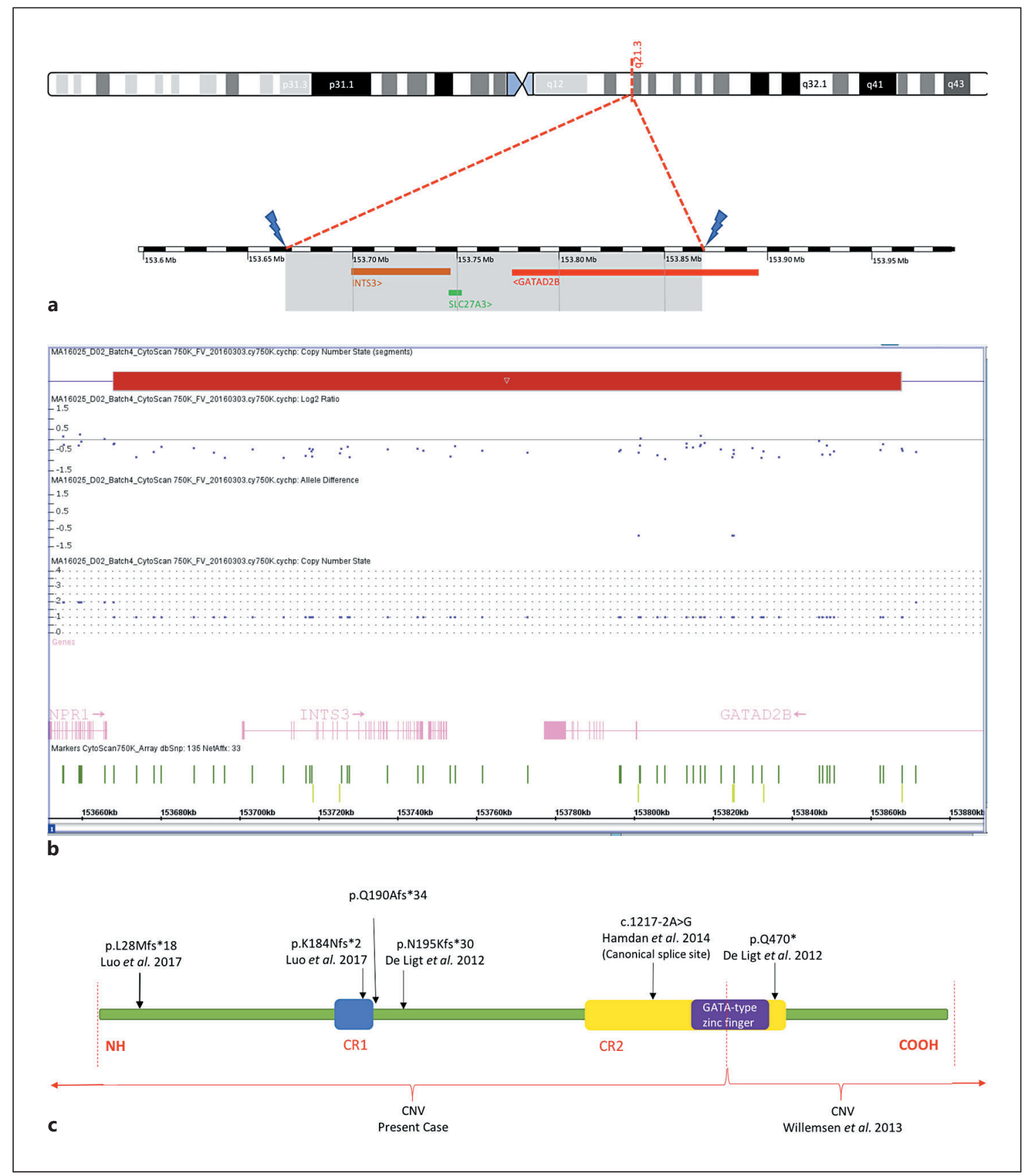

Fig. 3. a Location of the genes involved in the proband. CNV is located in 1q31.2 showing a complete deletion (gray) of the INTS3 (HI 24.77\%) and SLC27A3 (HI 62.33\%) genes. Additionally, we found a partial deletion (red) of the GATAD2B gene with an HI of $10.42 \%$, indicating a haploinsufficiency. HI, haploinsufficiency index. b Plot of $\mathrm{CNV}$ with $\log \mathrm{R}$ ratio and B-allele frequency. c Relative position of variants in the GATAD2B protein.

which was reported previously as pathogenic CNV related to MRD18 [Willemsen et al., 2013].

GATAD2B encodes a $\mathrm{p} 66 / \mathrm{GATAD} 2 \mathrm{~B}$ protein, as a part of the MeCP1 molecular complex (Fig. 4a). This complex is composed of 10 polypeptides including MBD2
(methyl-CpG-binding domain), $7 \mathrm{NuRD}$ (nucleosome remodeling and histone deacetylation) system components, and 2 polypeptides of $66 \mathrm{kDa}$ (p66a/GATAD2a) and $68 \mathrm{kDa}$ (p66/GATAD2B). NuRD is a macromolecular multiprotein complex composed of $1 \mathrm{CHD} 3$ or $\mathrm{CHD} 4$ 
Fig. 4. a MeCP1 multiprotein complex structure and $\mathrm{p} 66 \beta$ functions. The figure is composed of 10 polypeptides including MBD2, 7 NuRD system components, and p66a/p66 $\beta$ proteins. The CR1/CR2 domains of p $66 \beta$ (GATAD2B) are indicated; both are required for the correct association of MeCP1 complex and unmodified histone tail binding, respectively. b MeCP1 complex functions. It is a transcriptional regulator complex, during chromatin compacting and decompacting (chromatin remodeling), due to ATPase/deacetylase enzymatic activity. It recognizes and binds to Me-CpG DNA regions facilitated by the MBD2 protein.

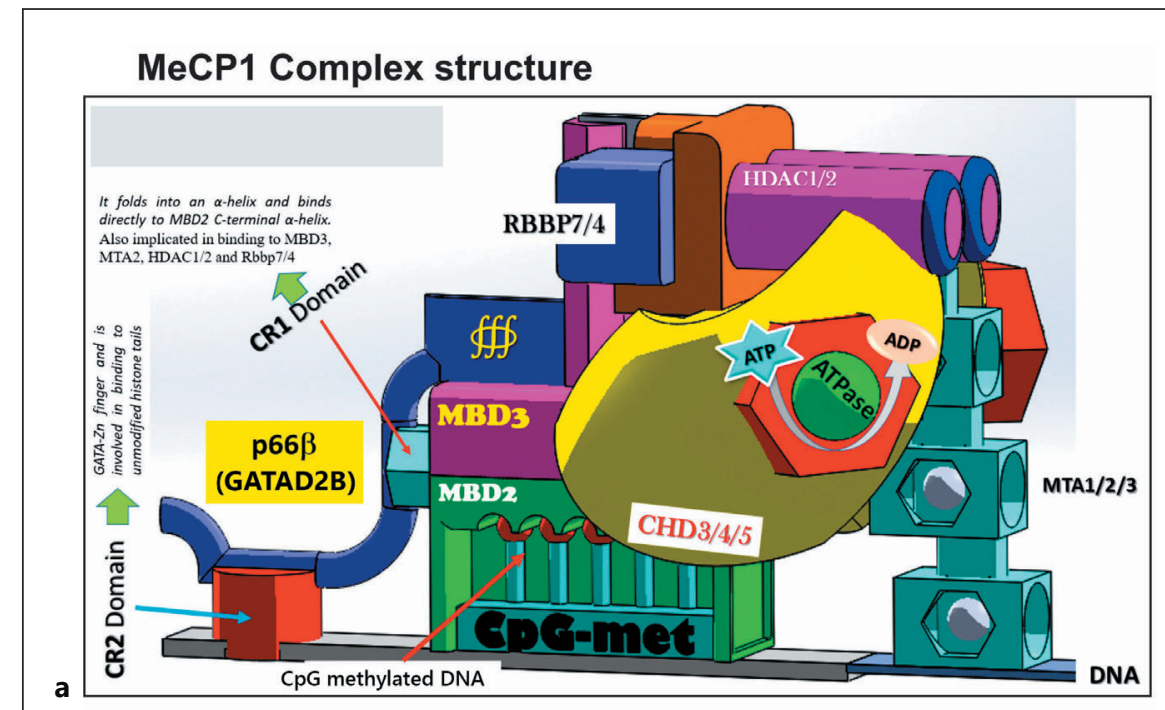

\section{MeCP1 Functions}

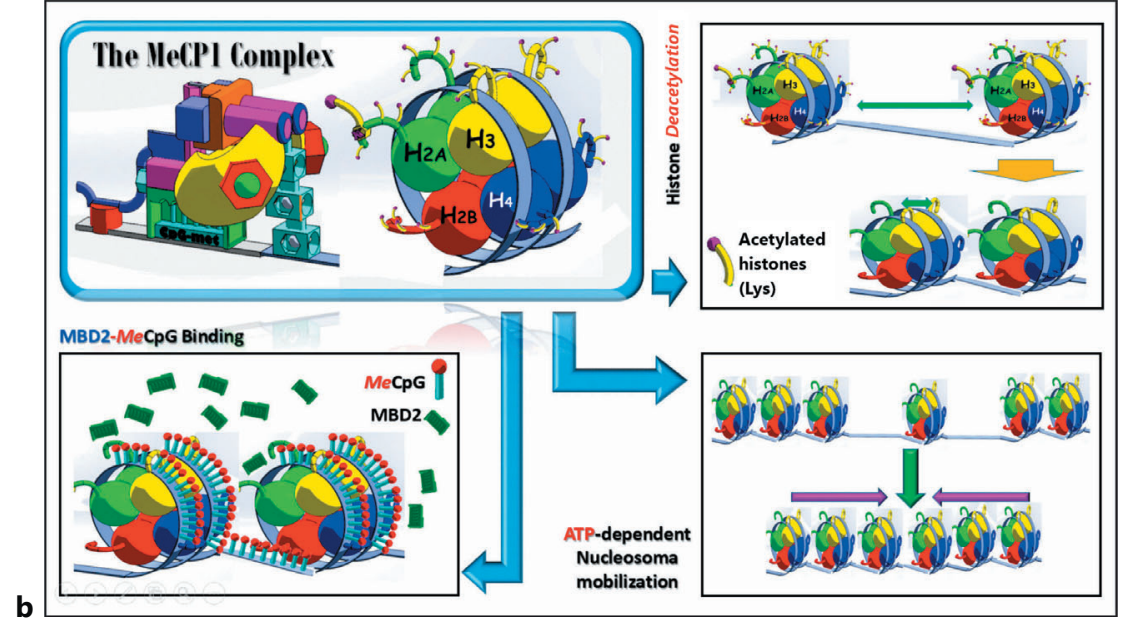

protein (chromodomain, helicase, DNA-binding domain), $1 \mathrm{HDAC} 1$ or HDAC2 protein (histone deacetylase core), 3 MTA1/2/3 proteins (metastasis associated), 1 MBD3 protein, $6 \mathrm{RbAp} 46 / 48$ proteins (retinoblastomaassociated protein), and 2 DOC-1 proteins (deleted in oral cancer). All of the abovementioned function as a transcriptional regulator complex by compacting and decompacting chromatin (chromatin remodeling) due to ATPase/deacetylase enzymatic activity [Torchy et al., 2015].

The MBD2 protein is necessary for the assembly of the MeCP1 complex (Fig. 4b) because it recruits NuRD components and directs them towards methylated DNA re- gions to form the MeCP1 complex. This complex, through preferential binding, remodeling, and deacetylating methylated nucleosomes in the presence of ATP, represses transcription by inhibition of transcriptional factors adhesion to the promoter and delays the RNA polymerase transcription process, hence leading to repression or silencing of methylated genes sequences [Feng and Zhang, 2001].

MBD2 acts as a scaffolding protein to drive p66a and p $66 \beta$ towards chromatin histone tails. The p66 proteins are transcriptional repressors due to histone deacetylase activity that contains 2 conserved regions (CR1/2). The CR1 region folds into the $\alpha$-helix and binds directly to 


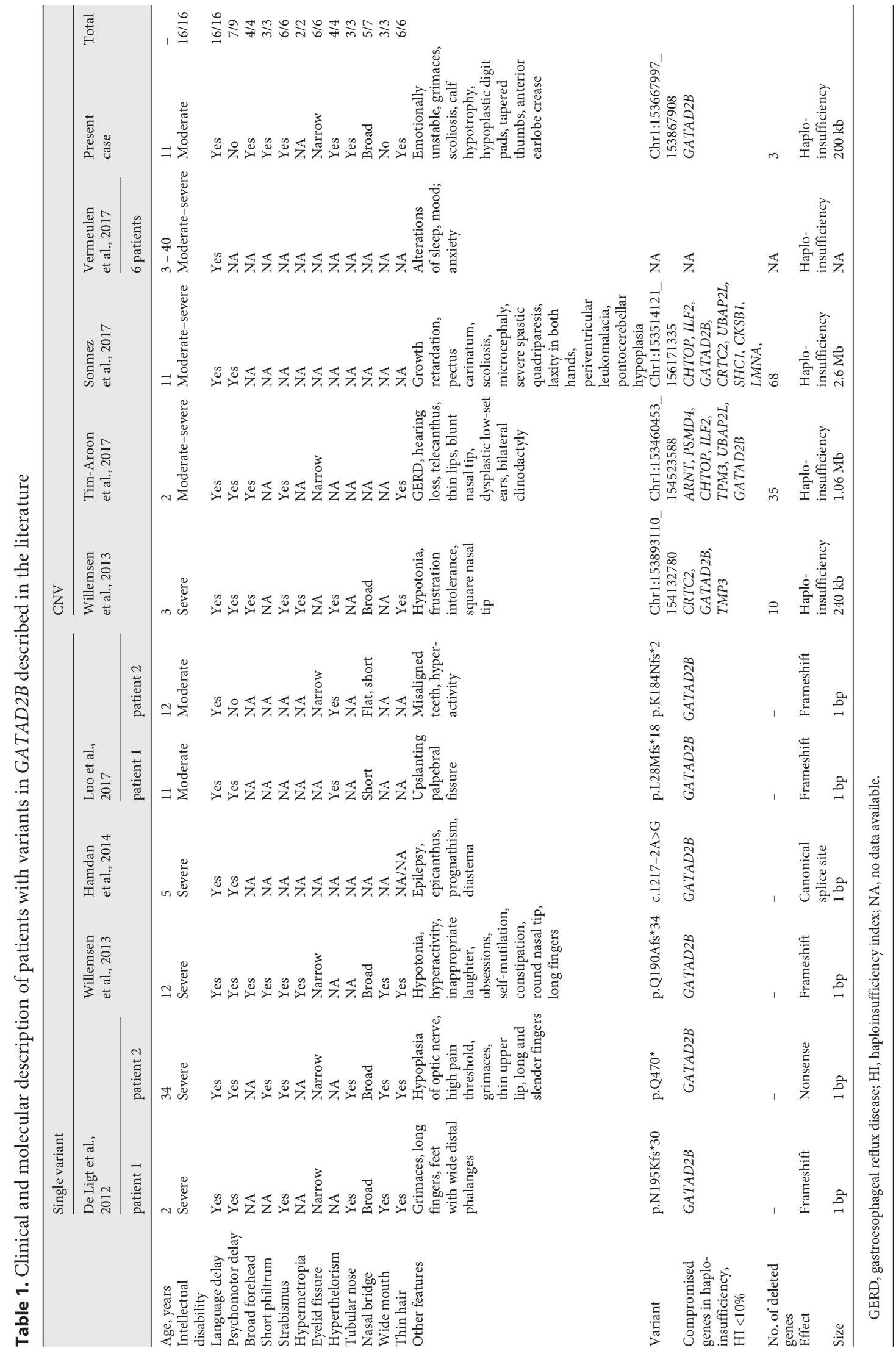




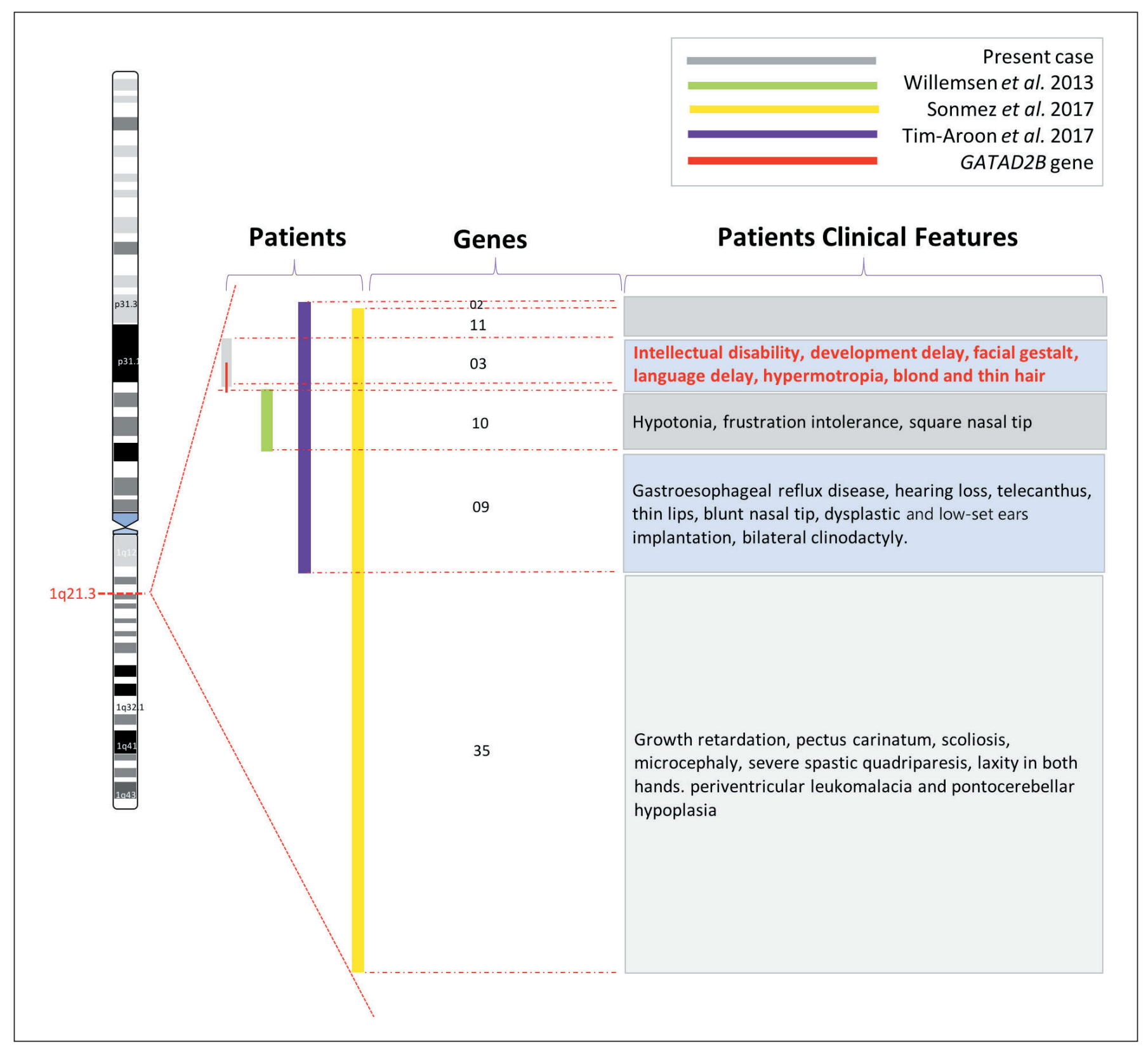

Fig. 5. Comparison of patients with CNVs in 1q21.3 in relation to clinical features and size of the deleted chromosome.

MBD2 C-terminal $a$-helix (also to MBD3, MTA2, $\mathrm{HDAC} 1 / 2$, and RBBP7/4), so 66 is required for the correct association of the $\mathrm{MeCP} 1$ complex. The $\mathrm{CR} 2$ region includes a GATA zinc finger domain involved in unmodified histone tail binding [Brackertz et al., 2002; Allen et al., 2013].

The $\mathrm{p} 66 \beta$ protein (GATAD2B) is highly and widely expressed in the brain, specifically in the amygdala, cor- pus callosum, cerebellum, caudate nucleus, hippocampus, and substantia nigra [Hirosawa et al., 1999].

The microdeletion found in this patient involves the INTS3, SLC27A3, and GATAD2B genes; however, only GATAD2B has been described. The GATAD2B gene has 11 exons, and in our patient, 10 exons are deleted. The conserved regions CR 1 and CR 2 are coded in exons 4 and $7-9$, respectively. The haploinsufficiency index reported 
in DECIPHER and ClinGen for GATAD2B was 10.42 and $3 \%$, respectively; hence, if the patient has only one functioning copy of this gene, it leads to a lower protein quantity and loss of function [Firth et al., 2009; Huang et al., 2010]. Also, the low concentration of the p66 protein may result in the reduction of the activity of the MECP1 complex. Evaluating dosage sensitivity for INTS3 and SLC27A3 shows haploinsufficiency indexes of 24.77 and $62.33 \%$, respectively, according to DECIPHER; however, ClinGen does not have any score yet. Nevertheless, INTS3 is part of the core complex together with human singlestranded DNA protein 1 which participates in DNA damage response and maintains genomic stability [Zhang et al., 2013; Vidhyasagar et al., 2018]. Furthermore, the SLC27A3 gene encodes one of the most relevant fatty acid transporter for supplying fatty acids to the fetal brain; our patient's variants were described in autism spectrum disorder (ASD) [Maekawa et al., 2015], although this case did not show clinical features of ASD.

To date, this female patient is the 16th case reported in the literature, of which 10 have CNVs and 6 single nucleotide variants (SNVs) (Fig. 4c). Patients with SNVs in GATAD2B have main clinical characteristics such as facial dysmorphism, grimaces, ID, development delay, poor speech, and hypotonia. Other psychopathological syndromes described are ASD, anxiety disorder, and sleep problems (Table 1). Unpublished additional clinical features related to patients with single variants in the GATAD2B gene were café-au-lait spots, calf hypotrophy, hypoplastic digits pads, scoliosis, anterior earlobe creases, and tapered thumbs. Comparing our clinical data with other CNV reports is more difficult because the number of compromised genes is variable (between 10 and 68) and dysmorphisms as well as the size of deletions of the 6 patients reported by Vermeulen et al. [2017] were not described. Nevertheless, the patients described in the literature have overlapping clinical features such as facial dysmorphism, ID, development delay, speech delay, hypermetropia, and thin blond hair (Fig. 5; Table 1) [Willemsen et al., 2013; Sonmez et al., 2017; Tim-Aroon et al., 2017; Vermeulen et al., 2017].

This variant has been reported as pathogenic and is consistent with the diagnosis of MRD18.

\section{Conclusion}

In this study, we emphasize the importance of applying modern molecular technologies, such as CMA, in order to delineate genotype-phenotype correlation, enabling precise diagnosis in patients with clinical features of broad genetic heterogeneity, such as ID, influencing the patient's medical treatment, prognosis, and risk recurrence and mainly when other routine tests are not sufficient for diagnosis. We describe a novel GATAD2B variant and expand the clinical spectrum of MRD18.

\section{Statement of Ethics}

The present study was performed following the ethical regulations of the Instituto Nacional de Salud del Niño (Lima, Peru). Written informed consent was obtained from the parents for publication.

\section{Disclosure Statement}

The authors declare no conflicts of interest.

\section{References}

Allen HF, Wade PA, Kutateladze TG: The NuRD de Ligt J, Willemsen MH, van Bon BW, Kleefstra architecture. Cell Mol Life Sci 70:3513-3524 (2013).

Brackertz M, Boeke J, Zhang R, Renkawitz R: Two highly related p66 proteins comprise a new family of potent transcriptional repressors interacting with MBD2 and MBD3. J Biol Chem 277:40958-40966 (2002).

Chiurazzi P, Pirozzi F: Advances in understanding - genetic basis of intellectual disability. F1000Res 5:F1000 Faculty Rev-599 (2016). T, Yntema HG, et al: Diagnostic exome sequencing in persons with severe intellectual disability. N Engl J Med 367:1921-1929 (2012).

Feng Q, Zhang Y: MeCP1 complex represses transcription through preferential binding, remodeling, and deacetylating methylated nucleosomes. Genes Dev 15:827-832 (2001).

Firth HV, Richards SM, Bevan AP, Clayton S, Corpas M, et al: DECIPHER: Database of Chromosomal Imbalance and Phenotype in Humans Using Ensembl Resources. Am J Hum Genet 84:524-533 (2009).
Hamdan FF, Srour M, Capo-Chichi JM, Daoud $\mathrm{H}$, Nassif C, et al: De novo mutations in moderate or severe intellectual disability. PLoS Genet 10:e1004772 (2014).

- Hirosawa M, Nagase T, Ishikawa K, Kikuno R, Nomura N, Ohara O: Characterization of CDNA clones selected by the GeneMark analysis from size-fractionated CDNA libraries from human brain. DNA Res 6:329-336 (1999).

Huang N, Lee I, Marcotte EM, Hurles ME: Characterising and predicting haploinsufficiency in the human genome. PLoS Genet 6:e1001154 (2010). 
Karam SM, Riegel M, Segal SL, Félix TM, Barros AJ, et al: Genetic causes of intellectual disability in a birth cohort: a population-based study. Am J Med Genet A 167:1204-1214 (2015).

Lazcano-Ponce E, Katz G, Allen-Leigh B, Magaña Valladares L, Rangel-Eudave G, et al: Intellectual development disorders in Latin America: a framework for setting policy priorities for research and care (in Spanish). Rev Panam Salud Publica 34:204-209 (2013).

Luo X, Zou Y, Tan B, Zhang Y, Guo J, et al: Novel GATAD2B loss-of-function mutations cause intellectual disability in two unrelated cases. J Hum Genet 62:513-516 (2017).

- Maekawa M, Iwayama Y, Ohnishi T, Toyoshima M1, Shimamoto C, et al: Investigation of the fatty acid transporter-encoding genes SLC27A3 and SLC27A4 in autism. Sci Rep 5: 16239 (2015).

Miller DT, Adam MP, Aradhya S, Biesecker LG Brothman AR, et al: Consensus statement: chromosomal microarray is a first-tier clinical diagnostic test for individuals with developmental disabilities or congenital anomalies. Am J Hum Genet 86:749-764 (2010).
Ropers HH: Genetics of early onset cognitive impairment. Annu Rev Genomics Hum Genet 11:161-187 (2010).

Sonmez FM, Uctepe E, Aktas D, Alikasifoglu M: Microdeletion of chromosome 1q21.3 in fraternal twins is associated with mental retardation, microcephaly, and epilepsy. Intractable Rare Dis Res 6:61-64 (2017).

- Srivastava AK, Schwartz CE: Intellectual disability and autism spectrum disorders: causal genes and molecular mechanisms. Neurosci Biobehav Rev 2:161-174 (2014).

Tim-Aroon T, Jinawath N, Thammachote W, Sinpitak P, Limrungsikul A, et al: 1q21.3 deletion involving GATAD2B: an emerging recurrent microdeletion syndrome. Am J Med Genet A 173:766-770 (2017).

Torchy MP, Hamiche A, Klaholz BP: Structure and function insights into the NuRD chromatin remodeling complex. Cell Mol Life Sci 72: 2491-2507 (2015).
Vermeulen K, de Boer A, Janzing JGE, Koolen DA, Ockeloen CW, et al: Adaptive and maladaptive functioning in Kleefstra syndrome compared to other rare genetic disorders with intellectual disabilities. Am J Med Genet A, E-pub ahead of print (2017)

Vidhyasagar V, He Y, Guo M, Talwar T, Singh RS, et al: Biochemical characterization of INTS3 and C9ORF80, two subunits of hNABP1/2 heterotrimeric complex in nucleic acid binding. Biochem J 475:45-60 (2018).

-Vissers LE, Gilissen C, Veltman JA: Genetic studies in intellectual disability and related disorders. Nat Rev Genet 17:9-18 (2016).

Willemsen MH, Nijhof B, Fenckova M, Nillesen WM, Bongers EM, et al: GATAD2B loss-offunction mutations cause a recognisable syndrome with intellectual disability and are associated with learning deficits and synaptic undergrowth in Drosophila. J Med Genet 50: 507-514 (2013).

Zhang F, Ma T, Yu X: A core hSSB1-INTS complex participates in the DNA damage response. J Cell Sci 126:4850-4855 (2013). 